\title{
Development and Validation of a New Risk Score for Infection From Coronavirus (SARS-COV-2) [Ri.S.I.Co], That We Learned on the Field Treating Coronavirus Disease (COVID-19) Patients
}

Paola Fugazzola ( $\triangle$ paola.fugazzola@gmail.com )

Ospedale Maurizio Bufalini https://orcid.org/0000-0002-6227-9276

Francesco Favi

Ospedale Maurizio Bufalini

Matteo Tomasoni

Ospedale Maurizio Bufalini

Claudia Zaghi

Ospedale Maurizio Bufalini

Chiara Casadei

Ospedale Maurizio Bufalini

Enrico Prosperi

Ospedale Maurizio Bufalini

Giacomo Sermonesi

Ospedale Maurizio Bufalini

Davide Corbella

Aziende Socio Sanitarie Territoriale Papa Giovanni XXIII

Federico Coccolini

Azienda Ospedaliero Universitaria Pisana

Beniamino Praticò

Ospedale Maurizio Bufalini

Vanni Agnoletti

Ospedale Maurizio Bufalini

Luca Ansaloni

Ospedale Maurizio Bufalini

Research article

Keywords: Sars-COV-2, COVID, diagnostic score, mass casualty

Posted Date: May 15th, 2020 
DOI: https://doi.org/10.21203/rs.3.rs-28815/v1

License: (c) (i) This work is licensed under a Creative Commons Attribution 4.0 International License. Read Full License 


\section{Abstract}

Background: The pandemic of Coronavirus Disease 2019 asked to change the organization of entire hospitals to try to prevent them to become epidemiological clusters. The actually adopted diagnostic tools are lacking of sensibility or specificity. The aim of the study is to create an easy-to-get risk score (Ri.S.I.Co., RIsk Score for Infection from new COronavirus), developed on the field, to stratify patients admitted to the hospital according to their risk of Covid-19 infection.

Methods: This prospective study included all patients who were consecutively admitted in the "suspected COVID-19 department" of the Bufalini Hospital, Cesena (Italy). All clinical, radiological and laboratory predictors were included in a multivariable logistic regression model to create a risk model. A simplified model was internally ed externally validated. Two score thresholds for stratifying the probability of COVID-19 infection were introduced.

Results: From 11th March to 5th April 2020, 200 patients were consecutively admitted. A Ri.S.I.Co lower than 2 had an higher sensibility than SARS-Cov-2 nucleic acid detection $(96,2 \%$ vs $65,4 \%, p<0,001)$. The presence of ground glass pattern at lung-CT scan had a lower sensibility than a Ri.S.I.Co lower than 2 $(88,5 \%$ vs $96,2 \%, p<0,001)$ and a lower specificity than a Ri.S.I.Co higher than $6(75,0 \%$ vs $96,9 \%, p<0,001)$.

Conclusions: We believe that the Ri.S.I.Co could allow to stratify admitted patients according to their risk, avoiding hospitals becoming themselves the main Covid-19 carriers. Furthermore, it could guide clinicians in starting therapies early in severe-onset cases with a high probability of COVID-19, before molecular SARS-CoV-2 infection is confirmed.

\section{Strengths and limitations of this study:}

Ri.S.I.Co., (RIsk Score for Infection from new COronavirus) is an easy-to-get risk-score developed on the field, to stratify patients admitted to the hospital according to their risk of Covid-19 infection.

We believe that the Ri.S.I.Co could allow to stratify admitted patients according to their risk, before molecular SARS-CoV-2 infection is confirmed, avoiding hospitals becoming themselves the main Covid19 carriers.

Ri.S.I.Co had an higher sensibility than SARS-Cov-2 nucleic acid detection and an higher sensibility and specificity of the presence of ground glass pattern at lung-CT scan.

Ri.S.I.Co was developed and validated on hospitalized patients, further studies would be necessary to understand if it is generalizable to non-hospitalized patients or on the population of other countries with different mean age, different prevalence of comorbidities and different health policies.

\section{Background}


Coronavirus (SARS-CoV-2) Disease (COVID19) (1)(2) has dramatically changed the world in last months, it shocked our lives, daily routine and relationships (3). As physicians, we had to react to the shock-wave: a large number of patients, whose access to Emergency Departments (ED) in a short period of time. Oftentimes this number rapidly filled up capacity of hospitals overwhelmed local and regional resources. This scenario has a simple, well-known but frightening name: it's a Mass Casualty Incident (MCl). Unlike common MCls (terrorist attacks, earthquakes, or accidents), Covid19 lasts for months.

In Western countries, Italy was in the frontline of Coronavirus (SARS-CoV-2) infection from last days of February, specially in northern regions (Lombardia, Emilia-Romagna, Veneto); what COVID-19 asked to health national systems was to change not only the type of working but also the organization of the hospitals: move and/or reduce surgical departments, theoretically "cleans", create beds for medical and / or intensive care units, theoretically "dirty".

This amount of patients with acute respiratory symptoms request that physicians and/or surgeons recycled themselves and temporarily neglected skills to adapt to the new situation.

This is even more important today in order to not expose patients and healthcare professionals to unnecessary biological risks, and to prevent hospitals themselves from being safe places to become themselves epidemiological clusters, as unfortunately happened in other realities (4).

"M. Bufalini" Hospital, Cesena Emilia-Romagna (Italy) is the HUB for Trauma, Stroke and Burns; it covers an area of about one million people, which increases dramatically during the summer season. During the first days of COVID-19 outbreak, one major problem was where and how managing patients suspected of SARS-CoV-2 infection. While laboratory tests were under evaluation, often patients needed treatment, even just oxygen, or closely medical monitoring. In our hospital Emergency Surgery Ward was identify to solve these problems.

Anamnestic criteria for suspected COVID-19 (at least one of: fever, cough, breathing difficulties, contacts with positive people and travel to endemic regions) lost their effectiveness: in a pandemic situation there are no safe people or places, suspicious symptoms covered the vast majority of patients in EDs. Blood samples showed a heterogeneous variability between normal and strongly altered. SARS-Cov-2 nucleic acid detection on upper respiratory tract specimen showed not sufficient sensibility and radiologic exams, chest X-ray but much more Thoracic High Resolution CT (HRCT), showed suspect, but not exclusive, signs related to interstitial involvement of the lungs.

While managing suspected COVID-19 patients on the field, we prospectively collected data with the aim of creating an easy-to-get risk score to meet our need to stratify and separate patients according to their risk of COVID19 infection; the goal should be to minimize the risk of intra-hospital SARS-CoV-2 infection and to ensure the best treatment available to everyone, even during emergency period.

\section{Materials And Methods}


We developed the new RIsk Score for Infection from new COronavirus (SARS-CoV-2) [Ri.S.I.Co] on a "construction sample" of consecutive patients admitted from 11th to 23th March 2020 in the "suspected COVID-19 department" of the Bufalini Hospital in Cesena (Italy). After excluding patients with missing data we internally validated the Ri.S.I.Co on the "internal validation cohort". Finally, we externally validated the Ri.S.I.Co on a "external validation cohort" derived from a "validation samples" formed by consecutive patients admitted to our department in a later time period (from $24^{\text {th }}$ March to $5^{\text {th }}$ April 2020).

\section{Construction sample}

This prospective study included all patients who were consecutively admitted from 11th March 2020 to 5th April 2020 in the "suspected COVID-19 department" of the Bufalini Hospital in Cesena (Italy). During this time period all patients who accessed the Emergency Department (ED) with fever higher than $37,5^{\circ} \mathrm{C}$ and/or respiratory symptoms (at the moment of presentation or in the last 10 days) or close contact with patients with a confirmed infection with the COVID-19 virus in the last two weeks, underwent a upper respiratory tract specimen collection (swab) for COVID-19 virus testing by RT-PCR (SARS-CoV-2 swab test). Patients who couldn't be discharged from the ED (with indication for clinical observation by the Territorial Medicine) and without the need of ventilatory support, were admitted to the "suspected COVID19 department".

After admission patients waited for a definitive confirmation or exclusion of an infection with the COVID19 virus.

Patients were treated for COVID-19 infection if he/she had almost one positive SARS-CoV-2 swab test or if he/she had strongly suspect radiological and clinical signs, even with negative swabs. All probable cases of COVID-19 infection with negative swabs, were reviewed by a final diagnosis committee composed of internal medicine specialists and/or pneumologists. If a patient was defined COVID-19 infected, he/she was transferred to a COVID-19 department or, in case of permissive conditions, discharged at home with indication for isolation and clinical observation by the Territorial Medicine. Otherwise, he/she was transferred to a non-COVID-19 department.

For the purpose of defining the outcome of the score, we considered a patient infected with the COVID-19 virus if he was treated for COVID-19 infection and/or he was transferred to a "COVID-19 department".

The following data were extracted for each patient: age, sex, contact with patients with a confirmed infection with the COVID-19 virus, vaccine status for the last influenza virus, symptoms other than fever and respiratory symptoms (joint or muscle pain, gastrointestinal symptoms, asthenia, headache, anosmia, ageusia), radiological findings in the thoracic HRCT report (presence of a ground-glass pattern, consolidations, interlobular septum thickening, pleural effusion, nodules, location of the pulmonary findings, presence of monolateral or bilateral findings), findings at the thoracic US performed in ED (presence of B-lines or subpleural consolidations), blood and urine tests performed in the ED (Legionella 
and Pneumococcus urinary antigens, Chlamydia and Mycoplasma serology, influenza A/B virus swab test, lymphocytes and neutrophils count, reactive $C$ protein (PCR), lactodehydrogenase (LDH), creatinfosfochinase (CPK), albuminemia, D-dimer, procalcitonin (PCT), troponin), respiratory rate (RR), oxygen saturation (sO2), walking test result and blood gas numbers at ED (p02/FiO2 ratio, p02, pCO2), COVID-19 swab tests results, treatment for COVID-19 virus infection, destination department/discharge. Any predictor recorded for $<50 \%$ of patients in the development data was not included in the modeling process.

After prospectively collecting the first 100 patients ("construction sample"), all clinical, radiological and laboratory predictors were included in a univariate analysis and then, after excluding non-significant factors, in a multivariable logistic regression model to create a risk score. We used multivariable logistic regression with backward stepwise selection with a $P$ value greater than 0.07 for removal of variables. We used the odd ratios $(\mathrm{OR})$ to estimate the coefficients associated with each potential risk factor to create a "regression model". A "simplified model", called Ri.S.I.Co, was constructed based on the regression coefficients which were approximated to coefficients directly proportional to the corresponding OR. The discriminating capacity of the simplified model was compared to the regression model through the average area under the ROC curve (AUC).

\section{Sample size}

All available data on the database were used to maximize the power and generalizability of the results. We did not perform a formal sample size calculation because there are no generally accepted approaches to estimate the sample size requirements for derivation and validation studies of risk prediction models. On the basis of some empirical investigations (5) we followed the widely adopted rule to have at least 10 outcome events per variable, or more precisely, per parameter estimated in the logistic regression.

\section{Internal validation}

We assessed internal validity calculating performance measures in the "internal validation cohort", derived from the "construction sample" after excluding patients with missing information on any of the predictors in the risk model. The average AUC of the score was calculated and compared with the performance measures of the most used diagnostic tests for COVID-19 infection: the first SARS-CoV-2 swab test and the thoracic HRCT.

\section{External validation}

The accuracy of the model was externally validated on an "external validation cohort", derived from a "validation sample" made of the prospectively collected data of 100 patients consecutively admitted in the "suspected COVID-19 department" in a later time period (from 24 $4^{\text {th }}$ March to $5^{\text {th }}$ April 2020). 
The "external validation cohort" derived from the "validation sample" after excluding patients with missing information on any of the predictors in the risk model. Similarly to the internal validation process, the average AUC of the score was calculated and compared with the performance measures of the most used diagnostic tests for COVID-19 infection: the first SARS-CoV-2 swab test and the thoracic HRCT.

Once a final model was defined, patients were divided into risk groups. Score thresholds for stratifying the probability of COVID-19 infection were introduced based on clinically acceptable sensibility and sensitivity.

\section{Construction and validation of a model without HRCT}

As many of the hospitals most affected by the pandemic in Northern Italy excluded thoracic HRCT from the diagnostic path of patients with suspected COVID-19 infection to save resources, we create a second simplified score ("Score without HRCT") on the construction sample excluding HRCT predictors from the multivariable logistic regression model. Subsequently we validated the model internally on the "internal validation cohort" and externally on the "external validation cohort", similarly to the model with HRCT.

\section{Statistical analysis}

Student's $t$ test and Fisher's exact test were used for analyzing differences in continuous variables and proportions. Logistic regression analysis was used in the multivariate analysis for the construction of the scores. The ROC area was used for comparing the discriminating capacity of the scores. Sensitivity and specificity of the new score were compared with the other diagnostic tests using McNemars' test.

This work was developed according to TRIPOD Statements (5).

\section{Results}

\section{Construction of the model}

From 11th March 2020 to 5th April 2020, 200 patients were consecutively admitted in the "suspected COVID-19 department" of the Bufalini Hospital in Cesena (Italy). Figure 1 reports participants flow diagram.

The first 100 patients formed the "construction sample". In Table 1 the characteristics of patients of the construction sample are reported with univariate analysis. Age lower than 60 years, LDH higher than 214 $U / L$, neutrophil count lower than $8 \times 10^{\wedge} 9 / L$, presence of ground glass pattern at thoracic HRCT, presence of bilateral findings at thoracic HRCT, contact with confirmed COVID infected patients and positive first SARS-CoV-2 swab were the significant risk factors for COVID-19 infection at univariate analysis. As SARS-CoV-2 swab is the accepted standard for COVID-19 diagnosis up to now, and all positive patients need isolated observation and/or treatment, we did not include this single item in the regression analysis. 
The significant risk factors for COVID-19 infection at multivariate logistic regression analysis are reported in table 2 with the corresponding regression score. These variables (age lower than 60 years, LDH higher than $214 \mathrm{U} / \mathrm{L}$, neutrophil count lower than $8 \times 10^{\wedge} 9 / \mathrm{L}$, presence of ground glass pattern at thoracic HRTC) and the relative OR made up the "Regression SCORE". A simplified score, Ri.S.I.Co, was constructed based on the regression coefficients which were approximated to coefficients directly proportional to the corresponding $\mathrm{OR}$.

The logistic regression analysis excluding HRCT finding is reported in table 2. These variables and the relative scores made up the "Regression SCORE without HRCT". A simplified "SCORE without HRCT" was constructed, similarly to the previous.

\section{Internal and external validation}

These two scores were internally and externally validated. The characteristics of patients in the internal and external validation cohorts are reported in table 1S. The discriminating capacity of the "Regression SCORE" (AUC 0,891, 95\%Cl:0,825-0,958) and of the simplified "Ri.S.I.Co" (AUC 0,892, 95\%Cl: 0,825-0,958) were very similar in the internal validation cohort. Likewise, the discriminating capacity of the "Regression SCORE without HRCT" (AUC 0,843, 95\%Cl: 0,762-0,924) and the "SCORE without HRCT" (AUC 0,830, 95\%Cl: 0,746-0,914) were very similar (Fig 1S).

The AUC of the Ri.S.I.Co calculated in the internal validation cohort was 0,892 (95\% Cl: 0,825-0,954), and it was significantly higher than the AUC of the presence of ground glass pattern at HRCT (AUC 0.701, $95 \% \mathrm{Cl}: 0,581-0,822)$. The AUC of the Ri.S.I.Co was not significantly different from the AUC of the first SARS-CoV-2 swab (AUC 0,933, 95\%Cl: 0,876-0,989) and of the "SCORE without HRCT" (AUC 0,830, 95\%Cl: $0,746-0,914)$. The ROCs and the relative AUCs of the internal validation cohort are reported in figure 2 and table 3.

The AUC of the RI.S.I.CO calculated in the external validation cohort is $0,754(95 \% \mathrm{Cl}: 0,640-0,868)$ and it was not significantly different form the AUC of the presence of ground glass pattern at thoracic HRCT (AUC 0,817, 95\% Cl: 0,721-0,913) and from the AUC of the first SARS-CoV-2 swab (AUC 0,827, 95\%Cl: $0,711-0,942)$. The AUC of the "SCORE without HRTC" was not significantly different from 0,5 . The ROCs and the relative AUCs of the external validation cohort are reported in figure 2 and table 3.

Table 4 reports the performance measures of the Ri.S.I.Co with 2 score thresholds $(>2$ and $>6)$ and for the maximum value of the score (9), of the first SARS-CoV-2 swab and of the presence of ground glass at thoracic HRCT.

The Ri.S.I.Co. $>2$ showed a higher sensibility than the first SARS-CoV-2 swab and than the presence of ground glass at thoracic HRCT (Ri.S.I.Co.: $96,2 \%$ vs first SARS-CoV-2 swab: $65,4 \%$, $p<0,001$ vs presence of ground glass at thoracic HRTC: $88,5 \%, p>0,001$ ), with a negative predictive value (PV-) of $95,5 \%$. 
The Ri.S.I.Co. $>6$ showed a higher specificity than the presence of ground glass at thoracic HRTC $(85,9 \%$ vs $75,0 \%, p<0,001$ ), with a positive predictive value (PV+) of the Ri.S.I.Co. $=9$ of $75,5 \%$ (higher than PV+ of the presence of ground glass at thoracic HRCT: 59.0\%). There was not significant difference between the specificity of Ri.S.I.Co. $>6$ and the specificity of the first SARS-CoV- 2 swab $(85,9 \%$ vs $100,0 \%, p=0,332)$.

Figure 3 reports the proposed Ri.S.I.Co and the relative risk groups.

\section{Discussion}

The explosion of COVID-19 on a global scale represents an epochal change in lifestyle, in personal relationships and puts the world medical and scientific community in front of a unique challenge; for the first time the whole of humanity is faced with a disease still largely unknown regarding virus itself, but also its pathophysiological damages and, more than all, its treatment.

None health-system, whether the single professional or the national and international health structures, can refrain from giving its contribution. In this scenario, some countries found themselves facing the emergency first, others had a few weeks to prepare and organize their defences. At present, however, despite the efforts of all, we still find ourselves having to chase a virus that runs faster than we do.

Within the pandemic declared by the WHO last March (6), the primary role turns out to be to identify early and certainly patients positive for COVID-19: to start as soon as possible experimental therapies and to isolate positives to effectively counteract the spread of virus transmission. The massive influx of patients suffering from respiratory symptoms, risked tilting, and sometimes had got, the emergency's nets of many countries, among the most developed in the world with national health systems considered at the top of the world. The solution was to change the face of hospitals, to make them suitable for the management of these patients: to create dedicated pathways, often having to use professional resources normally employed in other activities.

Why should surgeons handle with patients with a viral infection of respiratory tract? We, as Trauma and Acute Care Surgeons (TACS), believe that, as during others MCls, surgeons should be the first choice. Because TACS are used to manage many patients from EDs and to work closely with emergency physicians and/or ICU staff; they are prepared for these challenging clinical scenarios, and have the adequate skill to manage suspected COVID19 post-triage patients.

During exceptional periods, Acute Care Surgery's staff (both medical and nursing staff) is crucial: it can relieve the pressure on the EDs and take care of the post-triage management, also it being used to the management of complex surgical patients and their medical complications more than ultra-specialised surgical branches and certainly more inserted in the network relationships of modern hospitals, cause their daily routines (7).

Medical scores are widely used to help physicians in ranking patients by dubious medical diagnoses. Also Emergency Surgeons still face frequent with these clinical scenarios: an example is lower right 
abdominal pain, suspected for acute appendicitis, which represents one of the most frequent for which a surgical evaluation is required: in this clinical scenario the use of risk scores, such as the AIR Score, is of great use: by collecting clinical information and laboratory data it allows to group patients into subgroups at progressive risk of being affected by it (8).

COVID-19 erupted in early 2020 worldwide, clinicians are fighting against a new disease, largely unknown, and with a high virulence. Diagnostic tests are still perfectible and, even more seriously, not always available. Diagnosis of COVID-19 is still difficult; symptoms appear compatible with flu-like ones, different and aspecific. Regarding the epidemiological history of the patients, as contacts with positives or residence / travelling in endemic areas, in our opinion, the latter appear to be of limited utility: since the disease spread worldwide and because submerged portion of asymptomatic positives, thus distorting the aspect.

False-negative results in SARS-CoV-2 nucleic acid detection are caused by various reasons, such as the quality of the samples taken, the number of viruses and the stage of the disease. There are limited data on the rates of false-positive and false negative results for the various RT-PCR tests available. If a negative result is obtained from a patient with a high suspicion for COVID-19, additional specimens should be carried out, especially if only upper respiratory tract specimens were collected, as we do in our ED, prolonging the time needed for diagnosis (9).

Lung CT has been proposed as diagnostic tool, but its findings dependent on experience of radiologists and disease stage, and it needs specific "dirty" machines and results in more expensive and timeconsuming step.

The British Society of Thoracic Imaging (BSTI) recommends CT in seriously ill patients with suspected COVID-19 after chest x-ray is uncertain or normal (10). On the other side of the Atlantic Ocean, the American College of Radiology recommends CT for hospitalised, symptomatic patients with specific clinical indications, but radiological findings appears not to exclude a priori diagnosis (11).

Also, CT appears to be more specific in the later stages of COVID19, or on disease progression (12).

Ai and colleagues (13) showed that the sensitivity of chest CT in suggesting COVID-19 was $97 \%(95 \% \mathrm{Cl}$, 95-98\%, 580/601 patients) in positive RT-PCR samples, but 308/413 (75\%) patients with negative RT-PCR results, showed radiological findings.

The analysis of prospectively collected data of the first 100 patients admitted to our department and waiting for viral RNA RT-PCR confirmation, highlighted four significant risk factors for COVID-19 infection, which have become part of the Ri.S.I.Co.: age lower than 60 years, presence of ground-glass on lung-CT, an increased serum LDH and a normal neutrophil count. In order to maximize sensitivity e specificity of the score, we have identified two threshold values, allowing us to distinguish 3 groups of patients, with a progressive increase in the risk of being affected by COVID-19: low risk (Score 0-2), intermediate risk (Score 3-6), high risk (7- 9). 
The use of the Ri.S.I.Co. appears easy to use in daily clinical practice: it consists of 4 variables that are normally performed; even in the evaluation of CT only the radiological identification of the ground glass areas is necessary, without any measurements, or needing to evaluate multiple radiological parameters.

The variables used are normally dosed in any patient who accesses the PS, therefore widely reproducible.

In order to determine whether the Ri.S.I.Co. was advantageous, we compared their performance measures to the most adopted diagnostic tools for COVID-19 infection: the SARS-CoV-2 detection by RT-PCR in the first upper respiratory tract specimen collection (swab) and the presence of a ground glass pattern at thoracic HRCT.

Results showed that there was not significant difference between the AUC of Ri.S.I.Co., of the first SARSCOV-2 swab and of the presence of ground glass pattern at HRCT (Ri.S.I.Co: 0,754, 95\% Cl: 0,640-0,868; first SARS-CoV-2 swab: 0,827, 95\% Cl: 0,711-0,942; TC ground glass: 0,817, 95\% Cl: 0,721-0,913). However, although SARS-Cov-2 swab had a high specificity (100\%), its sensitivity was lower than a Ri.S.I.Co. lower than 2 (low risk group) $(65,4 \%$ vs $96,2 \%, p<0,001)$. The presence of ground glass pattern at HRCT had a lower sensibility than a Ri.S.I.Co. lower than 2 (low risk group) $(88,5 \%$ vs $96,2 \%, p<0,001$ ) and a lower specificity than a Ri.S.I.Co. higher than 6 (high risk group) $(75,0 \%$ vs $96,9 \%, p<0,001)$.

Because many hospital, likely for a policy of saving resources or for an inability to subject all patients to the examination, excluded lung CT as a diagnostic tool for COVID-19 infection, we create another score by eliminating the CT data; but, even if it performed well in the internal validation cohort, it loosed discriminating capacity in the external validation cohort. Therefore, in our opinion, lung CT is a necessary as one of the first diagnostic steps in the suspicion of coronavirus (SARS-CoV-2) infection.

Since most hospitals do not have a large number of single rooms or beds with a real insulation capacity, the stratification of patients according to the risk groups coming from the proposed Ri.S.I.Co, could be mainly used to separate admitted patients according to their risk. This could avoid low risk patients being infected from high risk patients and could avoid hospitals becoming the main Covid-19 carriers, as happened in many Italian realities. Furthermore, it could be a diagnostic tool additional to the lung-TC and to the SARS-CoV-2 detection by RT-PCR, increasing diagnostic sensibility and specificity.

During writing of the manuscript of this article, we read with interest a pre-print version of the COVID-19 early warning score (COVID-19 EWS) by colleagues from China (14): they retrospectively analyzed data from patients admitted for suspected COVID-19 (Training Dataset: 73 COVID+, 231 COVID-; Validate Dataset: 18 COVID+, 77 COVID-) and, as we did, they created an easy-to-get score for COVID-19 screening. The COVID-19 EWS included signs of pneumonia on CT, age older than 44 years, male gender, fever (in two different aspects: fever itself and fever more than $37,8^{\circ} \mathrm{C}$ ), presence of respiratory symptoms, history of contact with confirmed COVID-19 and neutrophil-to-lymphocyte ratio. Even if some risk factors were similar to our findings (sign of pneumonia on CT, neutrophil count), others were different. Among our construction sample the contact with a COVID-19 confirmed patients, the male gender, the presence of respiratory symptoms and the age older than 44 years were not significant risk factors at logistic 
regression analysis. We didn't consider fever in the analysis because fever and/or respiratory symptoms were the criteria for admission to our department. Applying the COVID-19 EWS score on our internal validation cohort, we found an AUC of $0,772(95 \% \mathrm{Cl} 0,668-0,877)$, a sensibility of $84,6 \%$ and a specificity $67,6 \%$. So, the performance measures of the COVID-19 EWS in our population were worse than the measures of Ri.S.I.Co. This fact could be explained in the different characteristics of the two population. First of all, our patients were older and the presence of fever and/or respiratory symptoms could be expression of other pathologies (cardiac or respiratory diseases). Among Italian population a lower number of upper respiratory tract specimen were collected than in China, without mass screening programs. This fact could reflect the lower weight of confirmed COVID-19 patients contact in predicting COVID-19 infection among our patients (many patients could have had contact with COVID-19 infected patients without knowing it). Furthermore, knowing the low sensibility of the SARS-CoV-2 detection by RTPCR in upper respiratory tract specimen, we didn't use it to define an infected patients. We included in the definition of "COVID-19 infected patient" also patients with negative SARS-CoV-2 swab, but with strongly suspect radiological and clinical signs, after a review by a final diagnosis committee composed of internal medicine specialists and/or pneumologists.

The limits of present study were the relatively small sample size and its observational nature. Being a study born to answer to our on-the-field need to stratify patients, we weren't able to take a longer period of time to collect a larger sample size to obtain an instrument that we needed immediately. Furthermore, present score was developed and validated only on hospitalized patients, further studies would be necessary to understand if it is generalizable to non-hospitalized patients or on the population of other countries with different mean age, different prevalence of comorbidities and different health policies.

\section{Conclusion}

The struggle against COVID-19 was, is and unfortunately will still be long. The validity of the diagnostic tests has yet to be fully confirmed; therefore, the physicians in next future should continue to consider as potentially infected patients with fever and/or respiratory symptoms. We believe that tools such as the Ri.S.I.Co could offer valuable help in the management of suspected patients while pending diagnostic confirmation. It could allow to separate admitted patients according to their risk, avoiding hospitals becoming the main COVID-19 carriers. Furthermore, it could guide clinicians in starting therapies early in severe-onset cases with a high probability of COVID-19, before molecular SARS-CoV-2 infection is confirmed.

\section{List Of Abbreviations:}

Ri.S.I.Co: RIsk Score for Infection from Coronavirus

COVID19: Coronavirus Disease

ED: Emergency Departments 
MCl: Mass Casualty Incident

HRCT: Thoracic High-Resolution Computer Tomography

PCR: reactive $C$ protein

LDH: lactodehydrogenase

CPK: creatinfosfochinase

PCT: procalcitonin

RR: respiratory rate

s02: oxygen saturation

OR: odd ratio

AUC: area under the ROC curve

95\%Cl: 95\% Confidence Interval

BSTI: The British Society of Thoracic Imaging

\section{Declaration}

\section{Ethics approval and consent to participate}

Because of the national and local regulations in force which prohibit any meeting to reduce the spread of the COVID-19 infection, it was impossible to get the study approved by our Ethics Committee. However, the study is purely observational in nature, the data were collected in an absolutely anonymous manner and the study was conducted in compliance with ethical principles originating from the Helsinki Declaration.

\section{Consent for publication}

Not applicable

\section{Availability of supporting data}

The datasets used and/or analysed during the current study are available from the corresponding author on reasonable request. 


\section{Competing Interests}

The authors declare that they have no competing interests

\section{Funding}

The authors received no specific funding for this work

\section{Authors' contributions}

P.F., F.F., M.T., L.A., D.C., F.C., V.A., B.P. contributed to the design and/or implementation of the research, P.F., F.F., M.T., C.Z., C.C., E.P., G.S., contributed to the collection of data, P.F., F.F., M.T., C.Z., L.A. contributed to the analysis of the results, P.F., F.F., L.A. to the writing of the manuscript. All authors revised and approved the final manuscript.

\section{Acknowledgments}

We really thank Alice Tani, Veronica Cappelli, Giorgia Bastoni and all the nursing and medical staff of the "AREA COVID IV piano" of the Bufalini Hospital in Cesena for the precious aid provided in data collection.

\section{References:}

1. Ren L-L, Wang Y-M, Wu Z-Q, Xiang Z-C, Guo L, Xu T, et al. Identification of a novel coronavirus causing severe pneumonia in human. Chin Med J (Engl). 2020;1.

2. Gorbalenya AE, Baker SC, Baric RS, de Groot RJ, Drosten C, Gulyaeva AA, et al. The species Severe acute respiratory syndrome-related coronavirus: classifying 2019-nCoV and naming it SARS-CoV-2. Nat Microbiol. 2020;5(March).

3. World Health Organization. Novel Coronavirus - China [Internet]. 2020, 12 January. Available from: https://www.who.int/csr/don/12-january-2020-novel-coronavirus-china/en/

4. Nacoti M, Ciocca A, Giupponi A, Brambillasca P. At the Epicenter of the Covid-19 Pandemic and Humanitarian Crises in Italy: Changing Perspectives on Preparation and Mitigation. NEJM Catal Innov Care Deliv. 2020;(Figure 1):1-5.

5. Moons KGM, Altman DG, Reitsma JB, loannidis JPA, Macaskill P, Steyerberg EW, et al. Transparent reporting of a multivariable prediction model for individual prognosis or diagnosis (TRIPOD): Explanation and elaboration. Ann Intern Med. 2015;162(1):W1-73.

6. World Health Organization. WHO Director-General's opening remarks at the media briefing on COVID19 - 11 March 2020. 2020. 
7. Catena F, Biffl W, De Simone B, Sartelli M, Di Saverio S, Kluger Y, et al. Emergency general surgeons: The special forces of general surgery (the "navy seals paradigm"). World J Emerg Surg. 2020;15(1):10-2.

8. Andersson M, Andersson RE. The appendicitis inflammatory response score: A tool for the diagnosis of acute appendicitis that outperforms the Alvarado score. World J Surg. 2008;32(8):1843-9.

9. World Health Organization. Laboratory testing for 2019 novel coronavirus (2019-nCoV) in suspected human cases. WHO - Interim Guid. 2020;2019(January):1-7.

10. British Society of Thoracic Imaging. Thoracic Imaging in COVID-19 Infection. 2020;(March):28.

11. ACR Recommendations for the use of Chest Radiography and Computed Tomography (CT) for Suspected COVID-19 Infection.

12. $X u X W, W u X X$, Jiang $X G, X u K J$, Ying $L J, M a C L$, et al. Clinical findings in a group of patients infected with the 2019 novel coronavirus (SARS-Cov-2) outside of Wuhan, China: Retrospective case series. BMJ. 2020;368(January):1-7.

13. Ai T, Yang Z, Hou H, Zhan C, Chen C, Lv W, et al. Correlation of Chest CT and RT-PCR Testing in Coronavirus Disease 2019 (COVID-19) in China: A Report of 1014 Cases. Radiology. 2020;2019:200642.

14. Song C-Y, Xu J, He J-Q, Lu Y-Q. COVID-19 early warning score: a multi-parameter screening tool to identify highly suspected patients. medRxiv. 2020;2020.03.05.20031906.

\section{Figures}

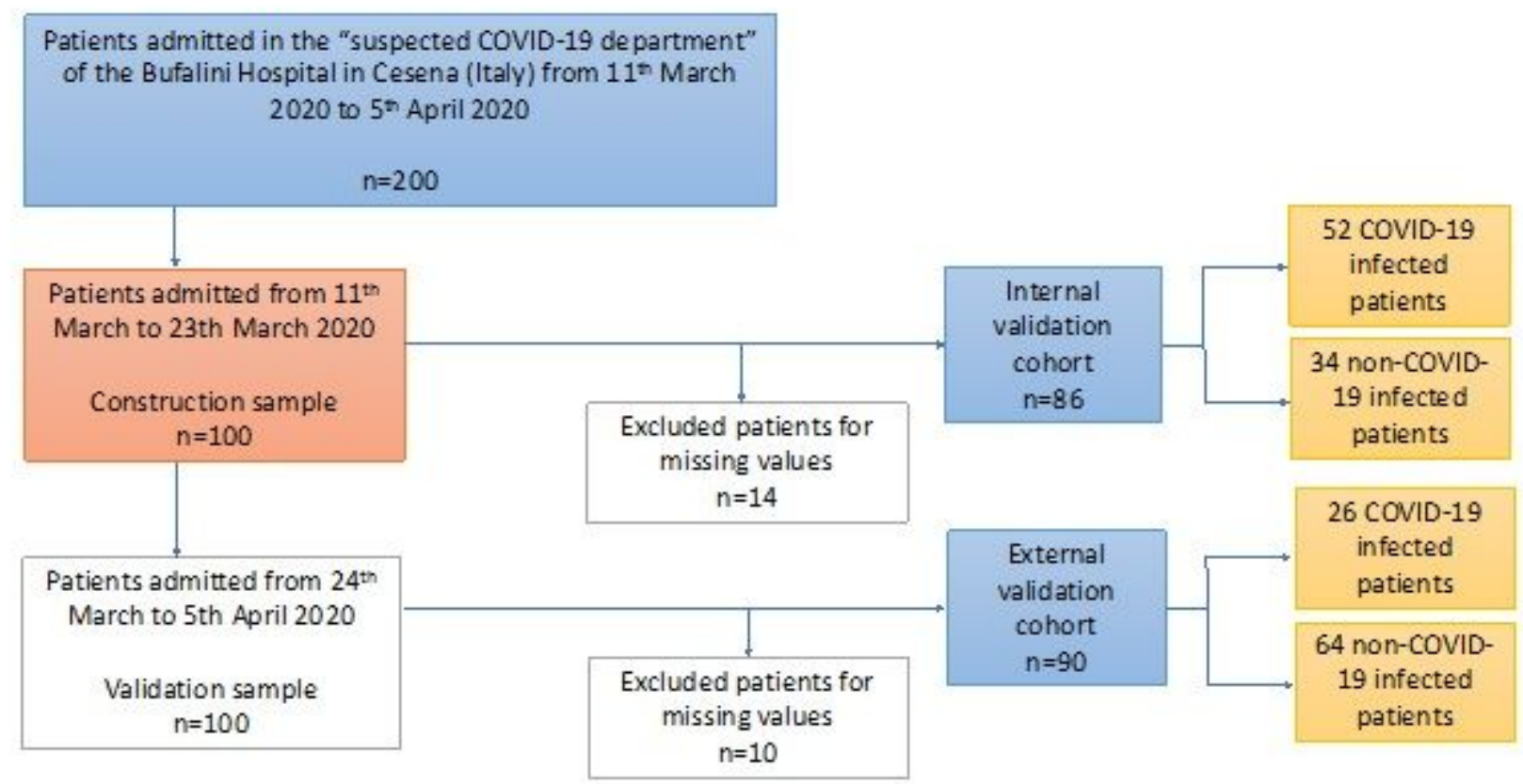

Figure 1 
Participant flow diagram

A)
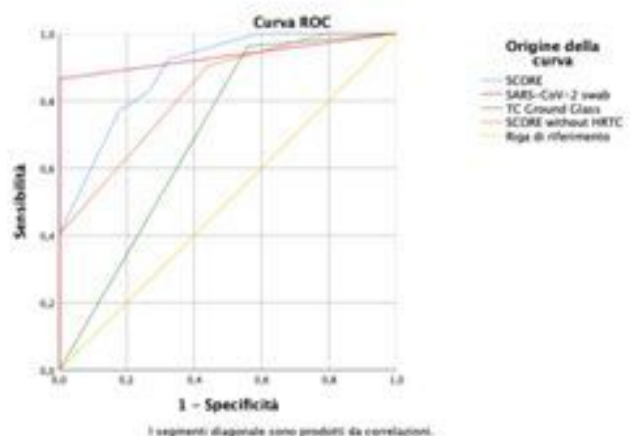

B)
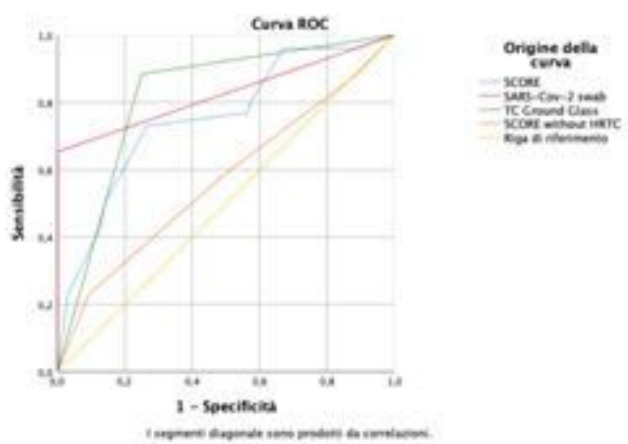

\section{Figure 2}

ROC of the internal validation cohort (A) and of the external validation cohort (B)

\begin{tabular}{cc}
\hline Varlable & SCORE \\
\hline HRCT Ground glass & 3 \\
Age $<60$ years & 1 \\
$\mathrm{LDH}>214 \mathrm{U} / \mathrm{L}$ & 2 \\
Neutrophil count $<8.00^{*} 10^{\wedge} \mathrm{g} / \mathrm{L}$ & 3 \\
\hline
\end{tabular}

\begin{tabular}{cc}
\hline RI.S.I.Co & RISK GROUPS \\
\hline $0-2$ & Low probability \\
$3-6$ & Intermediate group \\
$7-9$ & High probability \\
\hline
\end{tabular}

\section{Figure 3}

Scoring sheet with corresponding risk groups 


\section{Supplementary Files}

This is a list of supplementary files associated with this preprint. Click to download.

- Fig1S.docx 